\title{
A7 STRUCTURAL INSIGHTS INTO ANTIGENICITY OF RO/ LA RIBONUCLEOPROTEIN: SLE RELATED EPITOPES ARE CRYPTIC, MASKED BY HY1 RNA
}

J G Routsias, N C Kyriakidis, S Kotsakis, A G Tzioufas Pathophysiology Department, School of Medicine, University of Athens, Greece

\subsection{6/ard.2010.129577g}

Ro/La RNP is an antigenic ribonucleoprotein complex targeted by autoantibodies in patients suffering from rheumatic diseases such as Sjogren's syndrome (SS) and systemic lupus erythematosus (SLE). Human Ro60 structure has not been solved yet, but the structure of Xenopus laevis Ro60 has been determined. Two parts of the human La structure have been solved, one comprising the conserved La motif and its adjacent RNA recognition motif (RRM1) and another holding the C-terminal RRM2. For hY1 RNA, only its secondary structure has been experimentally determined on the basis of chemical and enzymatic RNA modification data. In our study the authors constructed in silico the overall structure of Ro/La RNP. Human Ro60 protein was built by homology modelling from Xenopus Ro, while $81 \%$ of human La was constructed by assembly of three parts: (1) 5-202 aa (La motif +RRM1), (2) 203-224 aa (linker region) and (3) 225-334 aa (RRM2). hY1 RNA model was built on the basis of its known secondary structure incorporating the regions from the solved Ro-RNA or La-RNA structures. Human Ro60, like Xenopus Ro60, is shaped like a doughnut with an inner hole. La possesses three consecutive RNA binding domains. Adjacent domains of La are arranged to face each other and form a V-shaped cleft of about $950 \AA 3$. hY1 RNA binds on the outside of the Ro60 doughnut and within the cleft formed between the La motif and an edge of the adjacent RRM1. hY1-RNA was found to completely mask epitopes 169-190 aa of Ro60 and 145-164 aa of La that were previously associated with SLE but not the SS related epitopes 211-232 aa of Ro60 and 349-364 aa of La. ELISA experiments with biotinylated synthetic hY1RNA, recombinant Ro60 and La antigens and purified antibodies against specific epitopes confirmed the above prediction. Thus, Ro60-hY1-RNA interaction was inhibited by anti-443-454, anti $211-232$ and anti $169-190$ antibodies by $13 \%, 45 \%$ and $76 \%$, respectively while La-hY1-RNA interaction was inhibited by purified anti349-364, anti-301-320 and anti-145-164 antibodies by $10 \%$, $43 \%$ and $97 \%$, respectively. Moreover, electrophoretic mobility shift assays demonstrated that Ro60 and La interactions with hY1 RNA can be inhibited by peptides corresponding to regions 169-190 aa and 145-164 aa of Ro60 and La autoantigens. Our study demonstrates that the SLE related epitopes on Ro and La autoantigens are actually cryptotopes in Ro/La RNP three dimensional structure masked by hY1 RNA. On the other hand SS related epitopes are directly accessible by autoantibodies in Ro/La RNP structure. 